\title{
CORRECTION
}

\section{Correction to: Aurora B kinase as a therapeutic target in acute lymphoblastic leukemia}

\author{
Hiroaki Goto ${ }^{1} \oplus$ - Yuki Yoshino ${ }^{1} \cdot$ Mieko Ito $^{1} \cdot$ Junichi Nagai ${ }^{2} \cdot$ Tadashi Kumamoto $^{3} \cdot$ Takesi Inukai $^{4} \cdot$ Yukari Sakurai $^{1}$. \\ Naoyuki Miyagawa ${ }^{1} \cdot$ Dai Keino $^{1} \cdot$ Tomoko Yokosuka $^{1} \cdot$ Fuminori Iwasaki $^{1} \cdot$ Satoshi Hamanoue $^{1} \cdot$ Masae Shiomi $^{1}$. \\ Shoko Goto ${ }^{1}$
}

Published online: 18 July 2021

(c) Springer-Verlag GmbH Germany, part of Springer Nature 2021

\section{Correction to: \\ Cancer Chemotherapy and Pharmacology \\ (2020) 85:773-783 \\ https://doi.org/10.1007/s00280-020-04045-9}

In the original publication of the article, two errors were found in Table 1 and Table 2 in the supplementary file. The corrected tables are given below:

Supplemental table 1; Characteristics of patients who provided samples originating newly established cell lines

\begin{tabular}{|c|c|c|c|c|}
\hline Cell line & $\begin{array}{l}\text { Age at sampling } \\
\text { /Gender }\end{array}$ & diagnosis & Karyotype & Fusion gene \\
\hline KCB2 & $1 \mathrm{y} 8 \mathrm{~m} / \mathrm{F}$ & BCP-ALL & $46, X X, t(8 ; 14)(q 24 ; q 32), \operatorname{add}(10)(q 22)$ & $\operatorname{lgH} / \mathrm{c}-\mathrm{Myc}$ \\
\hline KCB4 & $3 y 11 \mathrm{~m} / \mathrm{M}$ & BCP-ALL & $46, X Y, t(1 ; 3)(p 22 ; q 27)$ & ND \\
\hline KCB6 & $1 \mathrm{y} 9 \mathrm{~m} / \mathrm{M}$ & BCP-ALL & $\begin{array}{c}46, X Y, \operatorname{dup}(1)(\mathrm{p} 11 \mathrm{p} 32), \mathrm{t}(8 ; 14)(\mathrm{q} 24 ; \mathrm{q} 32) \\
\operatorname{der}(14), \mathrm{t}(1 ; 14)(\mathrm{q} 10 ; \mathrm{q} 10)\end{array}$ & $\operatorname{lgH} / \mathrm{c}-\mathrm{Myc}$ \\
\hline KCB7 & $14 \mathrm{y} 3 \mathrm{~m} / \mathrm{M}$ & BCP-ALL & $46, X Y$, del $(9)(p ?), t(14 ; 18)(q 32 ; q 21)$ & $\lg \mathrm{H} / \mathrm{Bcl} 2$ \\
\hline KCB9 & $17 \mathrm{y} 5 \mathrm{~m} / \mathrm{F}$ & BCP-ALL & 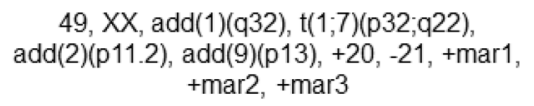 & MEF2D/Bcl9 \\
\hline KCB10 & $17 \mathrm{y} 0 \mathrm{~m} / \mathrm{F}$ & BCP-ALL & $47, \mathrm{XX},+\mathrm{r} 1$ & ND \\
\hline
\end{tabular}

Abbreviations; y: years, m: months, F; female, M; male, BCP-ALL: B cell precursor acute lymphoblastic leukemia, ND: not determined

The original article can be found online at https://doi.org/10.1007/ s00280-020-04045-9.

Hiroaki Goto

hgotou@kcmc.jp

Division of Hematology/Oncology, Kanagawa Children's Medical Center, 2-138-4 Mutsukawa Minami-Ku,

Yokohama, Japan
2 Department of Laboratory Medicine, Kanagawa Children's Medical Center, Yokohama, Japan

3 Department of Pediatric Oncology, National Cancer Center Hospital, Tokyo, Japan

4 Department of Pediatrics, School of Medicine, Yamanashi University, Kofu, Japan 
Supplemental table 2; names and concentrations of drugs used in the high throughput drug sensitivity test

\begin{tabular}{|c|c|}
\hline Drug & $\begin{array}{l}\text { Final concentration } \\
\text { (top dose) }\end{array}$ \\
\hline Dexamethasone ${ }^{1}$ & $100 \mu \mathrm{g} / \mathrm{ml}$ \\
\hline Clofarabine $^{6}$ & $100 \mathrm{ng} / \mathrm{ml}$ \\
\hline Eribulin $^{8}$ & $5 \mathrm{ng} / \mathrm{ml}$ \\
\hline SN382 & $20 \mathrm{ng} / \mathrm{ml}$ \\
\hline Cytarabine $^{2}$ & $100 \mu \mathrm{g} / \mathrm{ml}$ \\
\hline Etoposide $^{2}$ & $20 \mu \mathrm{g} / \mathrm{ml}$ \\
\hline L-Asparaginase ${ }^{4}$ & $10 \mathrm{U} / \mathrm{ml}$ \\
\hline Carboplatin" & $10 \mu \mathrm{g} / \mathrm{ml}$ \\
\hline Temozolomide $^{1}$ & $5 \mu \mathrm{g} / \mathrm{ml}$ \\
\hline Vinblastine $^{2}$ & $10 \mathrm{ng} / \mathrm{ml}$ \\
\hline Docetaxel| & $1 \mathrm{\mu g} / \mathrm{ml}$ \\
\hline Vincristine $e^{3}$ & $1 \mu \mathrm{g} / \mathrm{ml}$ \\
\hline 4-HO-CY2 & $40 \mu \mathrm{g} / \mathrm{ml}$ \\
\hline Mitoxantrone' & $500 \mathrm{ng} / \mathrm{ml}$ \\
\hline Topotecan ${ }^{7}$ & $500 \mathrm{ng} / \mathrm{ml}$ \\
\hline Ara-G hydrate & $88.5 \mu \mathrm{M}$ \\
\hline Fludarabine $^{3}$ & $5 \mu \mathrm{g} / \mathrm{ml}$ \\
\hline Azacitidine $^{2}$ & $1 \mu \mathrm{gg} / \mathrm{ml}$ \\
\hline Decitabine $^{3}$ & $1 \mu \mathrm{M}$ \\
\hline EPZ005687 & $1 \mu \mathrm{M}$ \\
\hline Tazemetostat ${ }^{3}$ & $1 \mu \mathrm{M}$ \\
\hline Vorinostat ${ }^{3}$ & $1 \mu \mathrm{M}$ \\
\hline Panobinostat $t^{3}$ & $1 \mu \mathrm{M}$ \\
\hline Olaparib ${ }^{3}$ & $1 \mathrm{\mu M}$ \\
\hline Linifanib $^{3}$ & $1 \mu \mathrm{M}$ \\
\hline Pazopanib ${ }^{3}$ & $1 \mu \mathrm{M}$ \\
\hline Sorafenib ${ }^{3}$ & $1 \mu M$ \\
\hline
\end{tabular}

\begin{tabular}{cc} 
Drug & $\begin{array}{c}\text { Final concentration } \\
\text { (top dose) }\end{array}$ \\
\hline Regorafenib $^{3}$ & $1 \mu \mathrm{M}$ \\
Lenvatinib $^{3}$ & $1 \mu \mathrm{M}$ \\
Lapatinib $^{3}$ & $1 \mu \mathrm{M}$ \\
Erlotinib $^{3}$ & $1 \mu \mathrm{M}$ \\
Crenolanib $^{3}$ & $1 \mu \mathrm{M}$ \\
Ibrutinib $^{3}$ & $1 \mu \mathrm{M}$
\end{tabular}

\begin{tabular}{cc} 
Drug & $\begin{array}{c}\text { Final concentration } \\
\text { (top dose) }\end{array}$ \\
\hline Saracatinib $^{3}$ & $1 \mu \mathrm{M}$
\end{tabular}

Saracatinib ${ }^{3} \quad 1 \mu \mathrm{M}$

${ }_{I C G-001^{3}} \quad 1 \mu \mathrm{M}$

Sonidegib ${ }^{3} \quad 1 \mu \mathrm{M}$

ABT-1993 $1 \mu \mathrm{M}$

$\mathrm{YM}^{155^{3}} \quad 1 \mu \mathrm{M}$

Tanespimycin $^{3} \quad 1 \mu \mathrm{M}$

Elesclomo $\beta^{3} \quad 1 \mu \mathrm{M}$

$\mathrm{PX}-4782 \mathrm{HCP}^{3} \quad 1 \mu \mathrm{M}$

2-Methoxyestradio ? $^{3} \quad 1 \mu \mathrm{M}$

Chrysin Dimethylether ${ }^{2} \quad 10 \mu g / \mathrm{ml}$

Quercetin $^{3} \quad 1 \mu \mathrm{M}$

RG-7112 3 1 1 M

$\mathrm{Ml}^{-773^{3}} \quad 1 \mu \mathrm{M}$

Bortezomib ${ }^{3} \quad 50 \mathrm{ng} / \mathrm{ml}^{3}$

Carfilzomib $^{3} \quad 1 \mu \mathrm{M}$

Z-LLNle-CHO $\quad 1 \mu \mathrm{M}$

RO4929097 $^{3} \quad 1 \mu \mathrm{M}$

GSK269962A $1 \mu \mathrm{M}$

$\mathrm{AZOT762}^{3} \quad 1 \mu \mathrm{M}$

$\mathrm{AZD}^{2} 208^{3} \quad 1 \mu \mathrm{M}$

Selisistat $\quad 1 \mu \mathrm{M}$

Apabetalone $^{3} \quad 1 \mu \mathrm{M}$

Pinometostat ${ }^{3} \quad 1 \mu \mathrm{M}$

GSK2879552 20 $\mathrm{HCl}^{3} \quad 1 \mu \mathrm{M}$

Linsitinib $\quad 1 \mu \mathrm{M}$

Palbociclib $^{3} \quad 1 \mu \mathrm{M}$

Abbreviations; 4-HO-CY: 4-hydroperoxy cyclophosphamide, HQPA: hydroxyquinazoline pyrazol anilide Drugs were purchased from 1: FUJIFLLM Wako Pure Chemical Co. (Osaka, Japan), 2: Funakoshi Co. (Tokyo, Japan), 3: Selleck Chemicals (Houston, TX) 4: Sigma-Aldrich Co. (St. Luis, MO), 5: Abcam (Cambridge, UK) 6: Adooq Bioscience (Irvne, CA), 7: Cayman Chemical (Ann Arbor, MI). 8: Funakoshi Co. and provision from Eisai Co. (Tokyo, Japan).

The original article has been corrected.

Publisher's Note Springer Nature remains neutral with regard to jurisdictional claims in published maps and institutional affiliations. 